\title{
APPROCHE COGNITIVE DE LA TRADUCTION ÉCONOMIQUE : RÉFLEXION THÉORIQUE ET RETOMBÉES PRATIQUES
}

\author{
Alessandra Rollo \\ alessandra.rollo@unisalento.it \\ Università del Salento
}

\section{Résumé}

Partant du constat que la traduction est à la fois un processus cognitif et linguistique, cet article veut s'interroger sur l'apport que l'approche cognitive peut donner à la recherche en traduction, en l'occurrence dans le domaine économique. Pour ce faire, nous nous appuierons sur quelques concepts clés élaborés dans le cadre de la Linguistique et de la Sémantique Cognitive (modèle de catégorisation prototypique, notion d'embodiment, scenes-and-frames semantics), l'objectif final étant d'élucider les activités cognitives sous-jacentes au mécanisme de conceptualisation et de formulation linguistique. En effet, encore que la traduction économique relève d'un domaine spécialisé, marqué par des traits de précision et de rigueur terminologique, elle n'est pas pour autant exempte des enjeux qu'implique toute activité traduisante. Le traducteur économique ne pourra donc que bénéficier d'une approche linguistico-cognitive, conjuguant l'axe lexical et la dimension sémantique.

\begin{abstract}
"Cognitive approach to economic translation: theoretical reflexion and practical effects"

Building on the assumption that translation is at the same time a cognitive and a linguistic process, the present article aims to investigate the contribution that the cognitive approach can make to translation research, especially in the economic domain. In order to account for the cognitive activities underlying the mechanism of conceptualization and linguistic formulation, we shall focus on some key concepts developed in the framework of Cognitive Linguistics and Semantics (model of prototype categorization, notion of embodiment, scenes-and-frames semantics). Indeed, even though economic translation comes under a specialized domain, marked by features
\end{abstract}


of exactness and terminological precision, it is not free from the problems which every translational activity entails. The economic translator can therefore profit from a linguistic-cognitive approach, integrating the lexical axis into the semantic dimension.

Mots clés : Traduction économique. Conceptualisation. Frame. Prototype. Contexte.

Keywords: Economic translation. Conceptualization. Frame. Prototype. Context.

Manuscript received on June 25, 2015 and accepted for publication on October 29, 2015. 


\section{Introduction}

Toute traduction est un acte communicatif complexe qui implique à la fois un processus cognitif et linguistique : il faut "interpréter pour traduire" (Seleskovitch \& Lederer 2001), c'est-à-dire appréhender et identifier le sens d'un message, puis le transférer dans d'autres schémas cognitifs et enfin le transposer dans un autre code linguistique. Il s'ensuit que la compétence linguistique est un élément indispensable mais pas suffisant, si elle n'est pas supportée par une compétence conceptuelle et intertextuelle, que ce soit dans le champ littéraire ou dans les domaines spécialisés.

C'est à partir de ce constat que nous allons focaliser notre attention sur la traduction économique, un domaine de spécialité en forte expansion dans la pratique professionnelle ainsi que dans les cursus des traducteurs, et pourtant fortement lacunaire du point de vue de la théorisation et de la recherche traductologique systématique (cf. Beuchat 2014: 23 sqq.). Nombreux sont encore les praticiens qui exercent leur métier se passant d'un modèle théorique, dans la conviction qu'une bonne compétence linguistique, une compréhension intuitive et une formation exclusivement pratique sont des atouts suffisants pour assurer la qualité des traductions. "Les questions théoriques ne sont pas abordées : elles semblent du domaine du superflu face aux problèmes urgents à régler par des professionnels qui recherchent d'abord une aide ponctuelle à la prise de décision" (Lavault-Olléon 2007 : 53).

Or, un éclairage théorique, appuyé sur une formation linguistique solide et une réflexion sur les processus mentaux de construction du sens, est fondamental pour accomplir au mieux la tâche traductive. André Clas (2005 : 4) remarque que "[t] out acte, toute décision, tout choix du traducteur a un soubassement théorique, même s'il reste inconscient, et une connaissance relativement encyclopédique". Sans prétendre modifier le statut du traducteur pour le rapprocher de celui du linguiste, l'inscription dans les programmes de formation des traducteurs de phénomènes qui déterminent la configuration et le fonctionnement du système linguistique, tels que le flou sémantique, la polysémie, la métaphore, etc., ne peut plus être marginalisée ; au contraire, une approche tenant compte de ces aspects peut s'avérer fructueuse 
et améliorer la qualité des traductions, surtout pour ce qui est de la traduction spécialisée qui pose notoirement beaucoup de problèmes aux professionnels (cf. Mejri 2005 : 122-125).

Dans le présent article, qui va s'inscrire dans une perspective cognitive, nous verrons comment la compréhension des mécanismes conceptuels sousjacents aux expressions langagières permet de mieux identifier certaines formes linguistiques et concourt à orienter les décisions traductionnelles. Les aspects lexicaux et terminologiques étant motivés, car étroitement liés aux problématiques cognitives et aux différents modes de conceptualisation d'une langue, le traducteur pourra opérer une sélection raisonnée dans l'éventail de traduisants possibles, parvenant aux solutions les plus pertinentes.

\section{Approche cognitive de la traduction}

$\mathrm{Au}$ cours des dernières décennies, les théories linguistiques se sont de plus en plus orientées vers des approches se plaçant sous le signe de la logique et de la cognition : linguistiques énonciatives, pragmatiques, cognitives; autant d'approches qui ont remis en question les axiomes traditionnels des courants formalistes (théorie componentielle du signifié, séparation entre sémantique et syntaxe, arbitraire des catégories formelles du langage, etc.). En particulier, la Linguistique Cognitive a contribué à jeter de la lumière sur les activités cognitives impliquées dans tout mécanisme de conceptualisation et de formulation linguistique (cf. Rollo 2008 : 54-55).

Ces études ne sont pas sans impact sur la pratique de la traduction, y compris la traduction spécialisée, car, comme le souligne Vandaele (2005: 418):

Lappréhension du sens dans un domaine de spécialité impose non seulement de prendre du recul à l'égard des unités lexicales prises indépendamment - cela n'est pas nouveau - mais aussi de comprendre les différents modes de conceptualisation mis en œuvre et la façon dont ils se révèlent dans les langues source et cible.

Avant d'entrer dans le vif du domaine qui nous intéresse ici, à savoir le domaine de l'économie et des affaires, il nous semble opportun d'introduire brièvement quelques concepts pivots de la Linguistique et de la Sémantique Cognitive, qui tiendront lieu de fil rouge pour une réflexion traductologique propédeutique à la pratique traductive.

Se rattachant à une tradition fonctionnaliste et anti-formaliste, la Linguistique Cognitive se configure comme une approche dynamique, holistique et expérientielle de l'étude de la langue et des motivations cognitives, 
préconisant une relation dialectique entre la faculté langagière et les autres aspects de la cognition humaine.

En vertu de sa fonction de catégorisation, le langage ne se limite pas à reproduire la réalité objective mais il lui impose une grille interprétative ; autrement dit, c'est une façon d'organiser notre connaissance du monde, dont le découpage en catégories sémantiques varie en fonction de notre vécu, de nos besoins et de nos expériences, qui diffèrent beaucoup selon les cultures. C'est là la position expérientialiste adoptée par les linguistes cognitifs (cf. Geeraerts \& Cuyckens 2007 : 5). Les catégories linguistiques ne sont que des catégories conceptuelles mises en mots au niveau lexical ou grammatical, "l'expression linguistique ne faisant que témoigner de la conceptualisation sous-jacente" (Vandaele 2005 : 428).

De plus, le processus de signification a des bases corporelles qui influent directement sur les structures de la pensée. Lun des concepts innovants de la Sémantique Cognitive est précisément celui d'embodiment ou "cognition incarnée", selon lequel l'esprit humain n'est pas une entité abstraite, mais il est incorporé à l'organisme qui interagit avec l'environnement extérieur et lui donne des perceptions sensorielles. L'esprit est donc conditionné par la dimension corporelle et biologique du parlant (propriétés perceptives et motrices) ainsi que par les lois du monde extérieur (comme la loi de gravité). Lidentification d'un fondement expérientiel de la pensée et du langage témoigne du principe de motivation sur lequel se fonde la langue en tant que système.

Tous ces aspects doivent être forcément pris en compte par un traducteur qui, lors du passage d'une langue à l'autre, est appelé à mettre en place une vraie réflexion au niveau cognitif et lexico-terminologique afin de retracer la carte conceptuelle ou mapping que sous-tend tel ou tel texte en ses éléments conceptuels constructifs (sémantisme des mots clés, relations logiques et sémantiques entre les concepts,...) en vue d'une correcte transposition (cf. Dancette \& Halimi 2005 : 554). Cela est d'autant plus vrai si l'on a affaire à des textes pragmatiques concernant des questions particulièrement importantes (tel est le cas des documents économiques ou juridiques, ayant souvent une visée décisionnelle), où la présence de contresens, de faux-sens ou de termes ambigus peut nuire à la signification globale du message et en compromettre la compréhension chez le public cible.

Le modèle de catégorisation privilégié par l'approche cognitive est le modèle prototypique - élaboré par la psychologue Eleanor Rosch et puis appliqué en linguistique - qui propose un paradigme alternatif plus souple et plus flexible par rapport à la rigidité du modèle classique des CNS (Conditions 
nécessaires et suffisantes), selon lequel il faut et il suffit qu'une entité possède les traits définitoires d'une catégorie pour y appartenir. Étant donné que la catégorisation n'est pas définie au moyen d'un critère unique, le paradigme du prototype permet de tracer des zones intermédiaires entre les différentes catégories et de classer les membres d'une même catégorie sur la base d'une ressemblance de famille. Par l'entremise de la notion wittgensteinienne de ressemblance ou air de famille, la version standard du prototype, envisageant que tous les membres d'une catégorie ont au moins un attribut en commun avec le prototype ou meilleur exemplaire du groupe, devient progressivement une version étendue ou polysémique, qui ouvre la voie à une conception multiréférentielle des catégories et s'applique aux phénomènes de sens multiple. Autrement dit, on conçoit qu'une catégorie regroupe les différentes acceptions d'un mot - et donc, les différents référents auxquels il renvoie ; au sein de la catégorie, tous les sens sont enchaînés entre eux par un ou plusieurs liens et sont décrits en termes d'effets prototypiques, à partir des sens premiers jusqu'aux sens périphériques (cf. Kleiber 1990 : 147 sqq.). De ce fait, le modèle prototypique s'avère plus adapté à organiser la gradation des concepts abstraits et des phénomènes pragmatico-lexicaux (comme la polysémie) pour lesquels il est impossible de définir une ligne de démarcation bien tranchée. La représentation des entités comme un ensemble de catégories discrètes cède ainsi la place à l'idée d'un continuum où l'on peut cerner des éléments plus ou moins saillants pour chaque catégorie.

$\mathrm{Au}$ nombre des notions utiles pour l'identification des éléments conceptuellement saillants, on peut compter le figure/ground alignment. Selon le principe perceptif du figure/ground que la Sémantique Cognitive a repris de la psychologie de la Gestalt, lorsque nous percevons un objet dans l'environnement extralinguistique, il nous apparaît comme un élément perceptuellement proéminent (figure ou "profil"), qui est mis en relief et se distingue du reste (ground ou "fond") (Talmy 1978). Le choix de l'élément saillant comme focus de notre attention est déterminé par des facteurs qui sont souvent d'ordre culturel, même si, à côté des perceptions subjectives propres à chaque individu, il y a des entités cognitivement et ontologiquement plus saillantes que d'autres, donc plus aptes à jouer le rôle de figure dans une configuration.

La théorie du figure/ground alignment s'inscrit dans le cadre de la scenesand-frames semantics de Fillmore (1977) : l'acte de compréhension d'un texte est déclenché par les mots du texte et les règles grammaticales qui les relient (linguistic frames), ainsi que par le bagage socioculturel et les expériences vécues que le récepteur a emmagasinés dans sa mémoire à long terme et qu'il 
réactive au contact de ces mots, de manière à construire des scènes cognitives ou mises-en-scène d'événements conceptuels (cognitive scenes). ${ }^{1}$

Pendant la phase de traduction, le texte de départ, élaboré par l'émetteur à partir de son répertoire de cognitive scenes, fournit par ses composantes linguistiques des frames qui évoquent à leur tour, dans l'esprit du traducteur-lecteur, une série de scènes cognitives composées d'éléments centraux (prototypiques) et marginaux (périphériques). Ces scènes reflètent la représentation du monde que se fait le traducteur en fonction de sa propre perception, de son expérience personnelle et de son modèle culturel de référence ; sur la base des scènes activées, il devra trouver des frames appropriés dans la langue d'arrivée de manière à recréer une gestalt (cf. Snell-Hornby 1995 : 81). Les frames construits sont d'autant plus semblables que les cultures d'appartenance sont plus proches; si la langue-culture source et la langue-culture cible ne partagent pas les mêmes modèles ni les mêmes scènes, le traducteur devra focaliser différemment les éléments évoqués et choisir un autre élément, qui soit prototypique dans la culture cible et donc lexicalisé.

Enfin, la notion de contexte situationnel, fortement réévaluée par les études cognitives, joue elle aussi un rôle essentiel dans l'élaboration des définitions sémantiques, impliquant une prise en compte tant des domaines où se manifestent les différences socioculturelles entre les diverses langues que des situations d'emploi professionnel. Il arrive souvent que le contexte modifie partiellement ou totalement le sens d'un terme ou d'une phrase ; le seul recours aux dictionnaires, sans tenir compte du contexte situationnel et du bagage culturel de chaque locuteur ou groupe de locuteurs, risque ainsi d'être insuffisant, voire fourvoyant, lors du choix de l'expression la plus convenable en langue cible.

En bref :

Il est $[\ldots]$ clair que la langue n'est pas inscrite dans les dictionnaires mais dans la tête de ceux qui la parlent. Dès lors, pour bien en comprendre la nature, il convient de se pencher sur l'univers conceptuel à partir duquel ces signes ont été formés (Delbecque 2002 : 31).

\section{La traduction économique dans une optique cognitive}

En nous inscrivant dans le sillage cognitif jusqu'ici retracé, nous allons maintenant aborder de plus près les spécificités de la traduction économique,

1. En fait, dans ses écrits suivants, Fillmore (1985) redéfinit la notion de frame, en lui reconnaissant à plein titre le statut de structure cognitive, "knowledge of which is presupposed for the concepts encoded by the words" (Fillmore \& Atkins 1992: 75). 
qui figure à l'heure actuelle parmi les spécialités les plus demandées dans le contexte globalisé, marqué par une intersection de la langue, de l'économie et de la mondialisation.

Rédacteur de milliers de documents qui facilitent la communication à l'échelle internationale, le traducteur économique est censé posséder avant tout une solide maîtrise des termes à forte technicité qui pointillent les textes à traduire, ainsi que des concepts qu'ils dénomment et des notions inhérentes; dans la pratique quotidienne, cela n'est pas une tâche aisée, vus l'ampleur et le dynamisme du domaine dont il est ici question (marketing, comptabilité, mécanismes économiques, opérations financières, boursières, etc.). Il faut également tenir compte, ainsi que précisé par Dancette (1992 : 199), que le domaine économique et commercial est caractérisé par "des échanges linguistiques subissant des influences complexes, [...] reflet de pratiques qui appartiennent à des systèmes culturels différents", ce qui explique l'extrême richesse et variété du lexique économique.

Etant donné son rôle de médiateur entre les langues et les cultures, le traducteur doit posséder les outils conceptuels et les connaissances lui permettant de mettre la langue pleinement au service de la société. La seule connaissance linguistique, le savoir-faire des mots, ne saurait toutefois suffire à faire d'un honnête professionnel un excellent traducteur. Il lui faut aussi une solide culture $[\ldots]$ une compétence fondée sur la polyvalence que les forces d'une économie désormais globale dictent au pigiste (Gémar 2007 : 34).

À côté d'une vaste compétence disciplinaire (ou thématique) et terminologique, le traducteur doit être tout aussi compétent pour ce qui a trait à l'architecture cognitive des langues mises en regard. D'un point de vue cognitif, les termes sont des unités conceptuelles représentant des nœuds de connaissance qui sont projetés linguistiquement au moyen d'éléments de nomination et qui constituent la structure conceptuelle d'un domaine spécialisé (cf. Cabré 2010 : 357). Cette définition va s'intégrer avec celle que proposent Vandaele et Raffo (2008: 15) : "les termes sont des dénominations spécialisées, c'està-dire des expressions linguistiques codées renvoyant à des catégories d'un domaine de connaissance, autorisant une économie de moyens au sein du discours".

Comme on le verra dans les pages qui suivent, il ne suffit pas de posséder une bonne connaissance des termes pour maîtriser un domaine de spécialité, mais il faut tenir compte de nombreux aspects de nature pragmatico-cognitive, tels les processus de catégorisation et de dénomination, les phénomènes de prototypie, les métaphores conceptuelles, esquivés par la théorie terminologique traditionnelle. Autant d'aspects encore peu développés dans les études de traduction économique et financière, qui abordent surtout, à partir 
d'une perspective cognitive, les questions liées aux métaphores conceptuelles et aux enjeux traductifs lors du transfert d'une langue à l'autre (cf. Serón 2005 ; Vinti 2006 ; Gallego 2010 ; Rollo à paraître).

\subsection{Différences de conceptualisation français-italien}

Il est de coutume de croire que les langues de spécialité sont soumises au principe de l'identité conceptuelle, en vertu du postulat terminologique, de matrice wüstérienne, préconisant une relation bi-univoque 1:1 entre signifiant et signifié : ${ }^{2}$ c'est le principe de l'isomorphisme ou de l'univocité invoqué par la terminologie classique qui s'inspire du crédo structuraliste et se situe dans une optique purement onomasiologique. En réalité, ce n'est pas toujours le cas, la plupart des termes recouvrant des notions assez vastes et ayant un sens influencé par le contexte, comme l'ont constaté certains terminologues qui, s'orientant vers une démarche sémasiologique, ont été conduits à remettre en question, ou à tout le moins, à atténuer la vision trop radicale de l'approche traditionnelle (cf. Thoiron \& Béjoint 2010 : 106-107).

La démarche cognitive met justement en évidence que le processus de dénomination est strictement lié au processus de conceptualisation : chaque terme correspondant à un concept laisse filtrer la façon de concevoir et d'interpréter la réalité propre à un groupe linguistique, quelles sont les propriétés considérées comme centrales et donc exprimées dans la communication, et lesquelles sont négligées. Emboîtant le pas aux sémanticiens cognitifs, Temmerman (2000) propose comme approche alternative pour la description terminologique la Sociocognitive Terminology, qui met l'accent sur l'interaction entre l'esprit humain, le monde extérieur (notre connaissance du monde est de nature expérientielle) et le langage, et reconnaît le potentiel cognitif de la terminologie dans les domaines spécialisés.

Sur le plan traductif, en raison d'une appréhension différente de la réalité, les termes de la langue source et ceux de la langue cible employés pour désigner une même notion peuvent s'avérer homologues, sans pour autant être identiques ou parfaitement équivalents, car ils éclairent des caractéristiques différentes du concept (cf. Béjoint \& Thoiron 1996 ; Dancette 1997).

En ce qui concerne la langue de l'économie et des affaires, il y a divers cas qui montrent une divergence d'éclairage au niveau terminologique entre

2. Il faut préciser que c'est là l'orientation générale de la théorie de la terminologie fondée par Wüster, qui a lui-même nuancé le principe de la bi-univocité, en admettant que c'était un idéal difficile, voire impossible à atteindre, surtout dans la langue générale, mais aussi dans les langues spécialisées. 
le français et l'italien. Prenons, par exemple, le terme français coût de la maind'œuvre (parfois appelé coût du travail ou prix du travail), correspondant en italien à "costo del lavoro" (plus fréquent que "costo della manodopera") : là où le français met en premier plan l'idée concrète de travail, en ce sens de labeur soutenu par les ouvriers, l'italien privilégie une représentation conceptuelle plus abstraite qui se réalise sur le plan verbal par l'emploi du générique "lavoro", indiquant toutes sortes de travail - une tâche, une activité, un emploi, la main-d'œuvre, un ouvrage, etc.

En italien, on utilise le terme "indennità di contingenza" (plus rarement "indennità di carovita"), traduit en français par indemnité de cherté de vie ou indemnité / allocation de vie chère (en droit, on parle d'allocation de raccordement), pour désigner une allocation temporaire accordée en présence d'une situation particulière, une petite intégration de la rétribution. Le terme "contingenza" est très fréquent dans le langage économique italien quand on se réfère à des phénomènes circonstanciels, à des situations ou à des conjonctures accidentelles; l'idée de contingence, en soi un peu nébuleuse, suggère quelque chose de passager, circonscrit à un laps de temps limité. Le français adopte une expression plus transparente, qui explicite le concept saillant, c'est-à-dire l'augmentation du coût de la vie.

On peut également trouver des termes qui ont des équivalents de traduction multiples selon le contexte d'emploi. Tel est le cas du terme prime, employé dans plusieurs syntagmes : prime à l'emploi ("incentivo all'occupazione"), prime d'encouragement ("incentivo"), prime de rendement / de productivité ("premio di rendimento / di produttività"), prime d'ancienneté ("premio di anzianità"), prime de licenciement ("indennità, contributo di licenziamento") en matière d'emploi ; prime de naissance ("premio alla nascita), prime de déménagement ("indennità di trasloco"), prime de réinstallation ("indennità di nuova sistemazione") en régime de protection sociale. On peut ici remarquer que les dénominations italiennes focalisent, selon le contexte, des traits spécifiques du concept désigné par prime : les termes "premio/incentivo" se rapportent à des situations positives, plus précisément "premio" est associé à une idée de récompense, "incentivo" à une forme de soutien et d'encouragement, alors que le terme "indennità" est utilisé dans des situations négatives (prime à titre de compensation ou de dédommagement).

Voici une autre unité terminologique : traitements et salaires. Dans le domaine de la finance, on a traitements et salaires ("stipendi e salari"), traitements et salaires étrangers ("redditi / emolumenti percepiti all'estero"), salaires et traitements en nature ("retribuzioni in natura"), celle-ci utilisée aussi en droit administratif ; pour ce qui à trait à la fiscalité, impôt sur les traitements 
et salaires ("imposta sui salari") ; en comptabilité et, en général, dans la vie économique, traitements et salaires bruts ("retribuzioni lorde") et traitements et salaires directs ("retribuzioni di base").

Pour l'italien, on peut mentionner le cas du terme "sconto", dont les différents usages et traduisants sont mis en lumière dans le tableau ci-dessous :

\begin{tabular}{|c|c|c|}
\hline IT & FR & DOMAINE \\
\hline sconto, deduzione & $\begin{array}{l}\text { escompte, retenue, précompte (réduction } \\
\text { du montant d'une dette à terme, } \\
\text { lorsqu'elle est payée avant l'échéance) }\end{array}$ & $\begin{array}{l}\text { FINANCES } \\
\text { (BANQUE) }\end{array}$ \\
\hline sconto (ribasso) & $\begin{array}{l}\text { rabais (réduction sur les prix de vente } \\
\text { accordée aux clients en raison d'un } \\
\text { défaut de qualité des produits ou de } \\
\text { non-conformité des produits avec la } \\
\text { commande), remise (réduction proposée à } \\
\text { un client sur un achat ultérieur) }\end{array}$ & $\begin{array}{l}\text { DROIT, ÉCHANGES } \\
\text { ÉCONOMIQUES ET } \\
\text { COMMERCIAUX }\end{array}$ \\
\hline sconto & escompte & COMPTABILITÉ \\
\hline scontare & $\begin{array}{l}\text { escompter (accorder un escompte, une } \\
\text { déduction d'une remise calculée selon le } \\
\text { temps restant à courir jusqu'à l'échéance } \\
\text { d'un effet de commerce) }\end{array}$ & $\begin{array}{l}\text { FINANCES } \\
\text { (BANQUE) }\end{array}$ \\
\hline $\begin{array}{l}\text { scontare (ridurre il } \\
\text { prezzo) }\end{array}$ & $\begin{array}{l}\text { rabattre le prix / sur le prix, rabattre } \\
\text { du prix une certaine somme (diminuer, } \\
\text { déduire, retrancher du prix) }\end{array}$ & DROIT \\
\hline $\begin{array}{l}\text { sconto, prezzo } \\
\text { scontato }\end{array}$ & $\begin{array}{l}\text { discompte, prix discompte, prix discompté } \\
\text { (système de commercialisation permettant } \\
\text { d'offrir au client des produits à prix } \\
\text { réduit) }\end{array}$ & VIE ÉCONOMIQUE \\
\hline $\begin{array}{l}\text { discount, magazzino } \\
\text { discount, magazzino } \\
\text { di sconto, discount }\end{array}$ & $\begin{array}{l}\text { magasin discount, magasin de rabais, } \\
\text { magasin (de) discompte, magasin } \\
\text { minimarge, minimarge }\end{array}$ & $\begin{array}{c}\text { ÉCHANGES } \\
\text { ÉCONOMIQUES ET } \\
\text { COMMERCIAUX } \\
\end{array}$ \\
\hline $\begin{array}{l}\text { analisi dei flussi } \\
\text { di cassa scontati, } \\
\text { metodo dei flussi di } \\
\text { cassa attualizzati }\end{array}$ & $\begin{array}{l}\text { méthode du flux monétaire actualisé, } \\
\text { méthode des flux de trésorerie actualisés }\end{array}$ & VIE ÉCONOMIQUE \\
\hline sconto, storno & $\begin{array}{l}\text { ristourne (réduction accordée dans le } \\
\text { cadre d'une transaction commerciale } \\
\text { sur le montant global des ventes d'une } \\
\text { certaine période: mois, trimestre,...) }\end{array}$ & $\begin{array}{l}\text { ÉCHANGES } \\
\text { ÉCONOMIQUES, } \\
\text { COMMUNICATION }\end{array}$ \\
\hline $\begin{array}{l}\text { sconto, riduzione, } \\
\text { detrazione, } \\
\text { abbassamento }\end{array}$ & abattement & $\begin{array}{l}\text { DROIT, SCIENCES } \\
\text { SOCIALES }\end{array}$ \\
\hline
\end{tabular}

Quelques exemples : 
1. It. Listituto di credito prevede uno sconto del $2 \%$ per i debiti saldati prima della fine dell'anno $=$ Fr. L'établissement de crédit prévoit un escompte de $2 \%$ pour les dettes payées avant la fin de l'année

2. It. Modalità di pagamento: in contanti con sconto del 3\%; uno sconto del $2,5 \%$ è previsto per ordini superiori a $€ 25000=$ Fr. Réglement $: \mathrm{au}$ comptant, avec un escompte de $3 \%$ et une remise de $2,5 \%$ pour toute commande dépassant $25000 €$

3. It. Gradiremmo sapere se poteste accordarci uno sconto del $4 \%$ sul prezzo di listino, in quanto i Vostri prezzi ci sembrano superiori a quelli della concorrenza $=$ Fr. Nous vous saurions gré de nous faire savoir si vous êtes disposés à nous accorder une remise de 4\% sur les prix-catalogue, puisqu’il nous semble que vos prix sont supérieurs à ceux de la concurrence

4. It. Ci scusiamo per l'inconveniente e vi accordiamo lo sconto del $35 \%$ che ci avete richiesto sull'importo della fattura $=$ Fr. Nous vous prions de nous excuser pour cet incident et nous nous empressons de vous accorder le rabais de 35\% que vous nous avez demandé sur le montant de la facture

5. It. Il fornitore dovrà concedere uno sconto / uno storno dell' $1 \%$ sugli acquisti di materie prime effettuati nel primo semestre dell'esercizio $=$ Fr. Le fournisseur devra accorder une ristourne de $1 \%$ sur les achats de matières premières effectués au cours du premier semestre de l'exercice

Le terme "sconto" évoque évidemment une pluralité de scènes cognitives, qui trouvent expression dans divers frames en français.

Comme le montrent les exemples qui précèdent, il est indispensable que le traducteur réfléchisse attentivement sur la dimension linguistique et cognitive d'un terme et qu'il en identifie le contexte d'emploi, avant qu'il ne procède au choix du bon traduisant, surtout quand il est confronté à des termes ayant un vaste spectre sémantique, avec des nuances insaisissables hors contexte. Si pour les termes complexes, comme prime à l'emploi, prime de licenciement, traitements et salaires bruts, etc., pour lesquels il existe déjà une équivalence codifiée, les dictionnaires spécialisés et les listes de collocations s'avèrent des alliés de taille en phase de traduction, il en va autrement pour les termes simples qui ne trouve pas de correspondance directe en langue cible et pour lesquels la gamme d'options traductives est assez large. Dans de tels cas, même les bases de données terminologiques et les glossaires spécialisés, tels que par exemple IATE ou le Vocabulaire de l'économie et des finances (2012), 
aussi complets soient-ils, ne peuvent pas être considérés comme un remède qui résout tout problème.

Dancette (1995 : 160) met en garde contre "l'illusion ou le piège de l'équivalent presse-bouton", puisqu'aucun dictionnaire ne peut prétendre à lui seul à l'exhaustivité terminologique. Il faut alors identifier l'organisation conceptuelle globale des domaines de connaissances dont relèvent les termes, l'interaction entre cognition et dénomination étant essentielle pour déterminer la signification exacte des termes et trouver les justes équivalents ; un travail scrupuleux, qui exige de la part du traducteur une grande expertise, une évaluation préalable du contexte et une activité systématique de consultation des ressources (y compris la recherche de documents authentiques sur le même domaine). ${ }^{3}$

Finalement la représentation conceptuelle peut être vue comme le point de convergence pour la traduction et le travail terminologique, un facteur clé dans l'élaboration des ressources terminologiques ${ }^{4}$ qui contribuent à l'acquisition des connaissances (cf. Faber \& Gómez-Moreno 2012 : 84).

\subsection{Langue de l'économie - langue générale - langues spécialisées : catégories communicantes}

La frontière entre langue générale et langue de spécialité est également assez vague, au point que l'on pourrait parler d'un continuum, une langue spécialisée se configurant comme "l'usage d'une langue naturelle pour rendre compte techniquement de connaissances spécialisées" (Lerat 1995 : 21) par des dénominations spécifiques au sein d'une communication spécialisée.

Tracer une limite entre langue générale et langue de spécialité est utile, ne serait-ce que pour circonscrire l'objet d'étude, mais tout indique que le mode de fonctionnement est semblable : comment pourrait-il en être autrement,

3. Deux dictionnaires méritent quand même d'être mentionnés, comme le rappelle Dancette (2003 : 154-155) : le Dictionnaire d'apprentissage du français des affaires et le Dictionnaire analytique de la distribution / Analytical Dictionary of Retailing, en tant qu'outils qui aident l'utilisateur à se construire un réseau sémantique et conceptuel autour du terme.

4. "Une recherche terminologique ne consiste pas uniquement à chercher un terme dans un dictionnaire bilingue ou une base de données afin de trouver son équivalent. Elle demande une analyse approfondie des systèmes et des concepts ainsi qu'une très bonne connaissance des règles linguistiques et des modes de formation des mots" (Lacroix 2010). Parmi les ressources textuelles, les corpus parallèles et les corpus comparables sont très utiles pour réfléchir sur l'organisation conceptuelle et sur la mise en forme du même type de matériel (fonction et situation communicative identiques ou similaires) dans des langues différentes. 
comment les mécanismes cognitifs pourraient-ils différer entre la vie quotidienne et l'activité scientifique? (Vandaele 2005 : 427).

La langue de l'économie, quant à elle, retient beaucoup de mots issus de la langue commune et soumis à une redéfinition sémantique : signification restreinte (spécialisation sémantique), glissement de sens ou sens nouveau (néosémie) ; employés dans un contexte spécialisé, ces mots activent des configurations conceptuelles propres au domaine en question. Voyons juste quelques cas à titre d'exemple.

Le verbe mondialiser, créé sur l'adjectif mondial, s'est implanté dans l'usage économique avec le sens de "prendre une dimension mondiale". Parallèlement, le couple global et globalisation, calqué sur l'anglais, s'est progressivement répandu, le terme globalisation, d'abord utilisé pour désigner une nouvelle configuration de la "mondialisation", s'étant désormais affirmé comme son synonyme ; d'où l'emploi de l'adjectif global (sens courant : "général", par ex. montant global = "importo complessivo") avec le sens de "mondial, planétaire", utilisé pour qualifier l'économie-monde. Litalien aussi adopte l'adjectif "globale" avec cette acception, même s'il tend à préférer l'emprunt anglais global : on traduit par exemple alter-mondialisme et alter-mondialiste par "movimento no global" et "no global".

Dans le terme complexe sincérité des comptes on retrouve le mot commun sincérité utilisé avec une acception technique, équivalant en italien à "veridicità di bilancio", où l'on emploie un terme qui relève d'un registre plus formel.

Le mot créneau, dont la traduction courante est "apertura, feritoia", nous offre un exemple de métaphore conceptuelle appliquée à la dénomination dans le domaine économico-commercial, en l'occurrence il désigne un segment de marché ("segmento, nicchia di mercato").

De même, il peut arriver que le lexique général soit contaminé par des termes spécialisés, dans une sorte d'aller-retour entre la langue générale et la langue spécialisée. Ainsi en est-il de termes comme inflation ("inflazione"), dont la signification originaire de "perte du pouvoir d'achat de la monnaie entraînant une augmentation générale des prix" se traduit dans le lexique commun en "dilatation, augmentation d'un phénomène, répétition excessive d'un argument", ou bien, actions à la hausse / à la baisse ("azioni in rialzo / in ribasso"), que l'on utilise dans le domaine économico-financier et juridique, mais aussi pour parler de la réputation de quelqu'un.

En plus de cela, il est à noter que la limite entre les vocabulaires spécialisés n'est pas si tranchée, les différents domaines étant souvent reliés par des échanges réciproques. Comme le rappelle Resche (2002:13) : 
Il s'avère que la tendance à la pluridisciplinarité, les progrès rapides en matière de recherche, et les échanges facilités par les technologies nouvelles ont conduit à un décloisonnement des domaines. Les domaines spécialisés [...] ne sont plus imperméables, les frontières s'estompent et les terminologies qui sont l'expression des notions ne sont pas des monolithes.

Dans plusieurs textes produits dans le domaine des affaires, le lexique économique s'entrecroise avec le langage politique ou juridico-administratif (contrats, différends, droit des sociétés, politiques économiques internationales, ...), ce qui implique un effort majeur pour comprendre la terminologie utilisée et restituer le sens exact dans la langue d'arrivée.

En voici quelques exemples :

6. Fr. Règlement extrajudiciaire des différends internationaux = It. Risoluzione stragiudiziale delle vertenze (controversie) internazionali

7. Fr. Sentence arbitraire = It. Lodo, sentenza arbitrale

8. Fr. Droit (impôt) de douane / d'accise / fiscal / de succession / de timbre= It. Dazio doganale / accisa (imposta di consumo) / diritto fiscale (tributario) / tassa di successione (diritto successorio / di successione) / imposta di bollo

9. Fr. Action libérée / partiellement libérée = It. Azione liberata / non interamente versata

10. Fr. Société coquille = It. Shell company (Conchiglia societaria vuota)

11. Fr. Société qui vole au secours d'une autre société menacée d'un raid / d'une OPA = It. Cavaliere bianco (White Knight) in difesa di un proprietario minacciato da un raid / dalla scalata di un'altra società

12. Fr. Action de groupe = It. Class action

13. Fr. Détaxe = Duty free (ou "esonero fiscale, franchigia doganale")

14. Fr. Les recettes affectées externes devraient faire l'objet d'un report de droit $=$ It. Le entrate con destinazione specifica esterne dovrebbero essere riportate automaticamente

On peut constater, entre autres, que l'italien assimile beaucoup plus la terminologie anglaise, contrairement au français, qui opte davantage pour la néonymie traductive (néoformations) et le recours aux calques.

\subsection{Le modèle prototypique : catégorisation et traduction des termes polysémiques}

Il paraît évident qu'à l'instar des catégories humaines, qui ne présentent pas de contours rigides, les catégories conceptuelles et linguistiques ne constituent pas de compartiments étanches, définis en termes absolus, vu qu'il y a 
des zones d'indétermination et de chevauchement qui laissent entrevoir une sorte de continuité.

Reposant sur l'identification des propriétés saillantes d'un concept ou d'une entité, le modèle prototypique, qui est à la base de la conception sémantico-cognitive du lexique, représente un instrument précieux pour la définition d'un terme, spécialement des termes polysémiques qui, de toute évidence, ne sont pas bannis du domaine de l'économie. En effet, quoiqu'on revienne souvent sur les caractéristiques de monosémie stable, d'univocité, de définitions précises et d'un sens uniquement référentiel, en tant que marques spécifiques de la terminologie qui caractérise les langues de spécialité, "les termes, comme les mots, tendent a la polysémie à partir du moment où ils s'insèrent dans des discours - ce qui est quand même le cas de la plupart d'entre eux" (Thoiron \& Béjoint 2010 : 107).

L'un des traits distinctifs de la Sémantique Cognitive réside dans l'assertion qu'il n'y a pas de séparation nette entre sémantique et pragmatique, entre signifié linguistique conventionnel et interprétation contextuelle, mais il existe plutôt un signifié encyclopédique qui s'actualise dans différents contextes d'emploi. Il incombe au traducteur, le cas échéant, de dégager les différents sens d'un terme, de détecter le sens plus central ou prototypique par rapport à d'autres qui sont plus marginaux ou périphériques, et de choisir l'équivalent approprié.

Soit le mot portefeuille, dont le sens premier de "serviette, enveloppe" fournit le point de départ pour différentes significations, des plus générales aux plus spécifiques, partageant des éléments de sens commun. Le mot peut désigner :

a. une enveloppe, un étui d'étoffe ou de cuir, servant à renfermer des papiers

b. un cartable ou une serviette de cuir, muni(e) de poches et de compartiments, qui sert à garder et à porter des papiers, des livres, etc.

c. une pochette, le plus souvent de cuir, d'assez petite dimension, où l'on range billets de banque, papiers, cartes de visite, etc. (synonyme: porte-monnaie)

d. (Politique) (figuré) le titre, les fonctions de ministre (le portefeuille des Affaires étrangères)

e. (Finance) l'ensemble des effets de commerce et des valeurs mobilières détenus par un individu, une banque ou une entreprise (portefeuille titres ; portefeuille cambiaire)

f. une collection de dessins ou d'estampes (le portefeuille d'un peintre). 
Les traduisants italiens sont "custodia, astuccio" pour a., "cartella, borsa portadocumenti" pour b., "portafoglio" pour les acceptions c., d., e. (sens littéral pour c., sens figuré par extension métaphorique pour d. et par extension métonymique pour e.), "collezione, raccolta" pour $\mathrm{f}$.

15. Fr. Le nouveau Ministre sans portefeuille = It. Il nuovo Ministro senza portafoglio

16. Fr. Les portefeuilles des fonds de pensions peuvent constituer une remarquable contribution à la stabilité du marché financier = It. I portafogli dei fondi pensione possono costituire un notevole contributo alla stabilità del mercato finanziario

On peut voir que les sens multiples d'un mot se distinguent par une différence de prééminence, tout en restant reliés par des liens systématiques (processus de généralisation et/ou de spécialisation, relation métaphorique de ressemblance, relation métonymique de contiguité) ; tout cela peut être représenté sous forme de réseau radial, organisé autour d'un certain nombre de traits prototypiques.

Nous reprenons ici la configuration des sens du mot canard, polysémique en français, telle qu'elle a été illustrée par Delbecque (2002 : 55-57), et nous y ajoutons l'acception que le mot acquiert dans l'expression canard boiteux employée dans le domaine économique pour parler d'une entreprise peu solide, désormais improductive ; en italien, l'expression correspondante, démétaphorisée, est "impresa malandata, in cattivo stato, non più produttiva".

Extension de la signification de canard (du sens premier aux sens périphériques) :

$$
\begin{aligned}
& \rightarrow \text { (Métonymie }) \\
& \rightarrow \text { (Métaphore }) \\
& \rightarrow \text { (Spécialisation }) \\
& \rightarrow(\text { Généralisation }) \\
& \rightarrow \text { (Métaphore/Spécialisation }) \\
& \rightarrow \text { (Spécialisation) }
\end{aligned}
$$

a. "oiseau aquatique"

b. "viande de cet oiseau"

c. "fausse nouvelle" (lancée dans la presse)

d. "journal"

e. "fausse note"

g. "le moins beau"

h. "entreprise instable" (trébuchante comme un canard)

f. "morceau de sucre"

5. L'expression littérale "anatra zoppa", calque de l'anglais lame duck, est répandue exclusivement dans le jargon journalistique et politique italien pour désigner le Président des États-Unis à son second mandat sans majorité au Congrès et, par extension, un politicien qui, en dépit de sa charge institutionnelle, n'est pas en mesure d'exercer son pouvoir. 
Réseau radial des sens de canard:

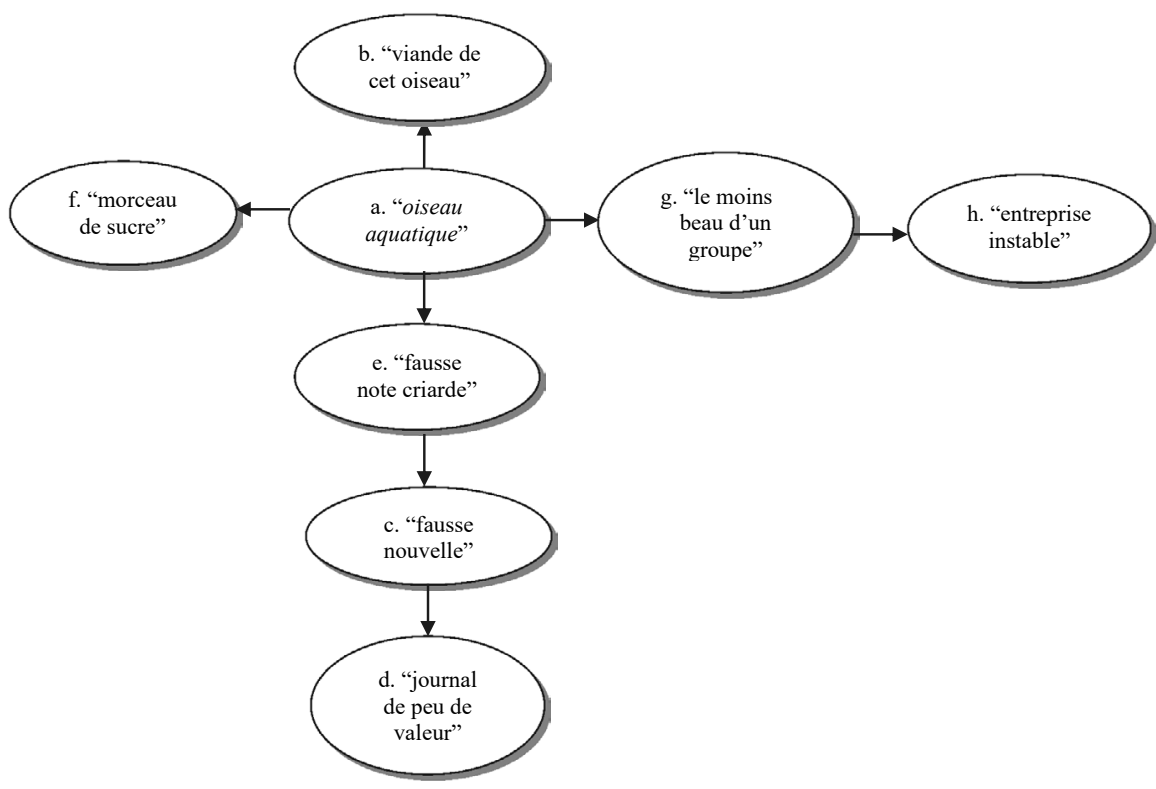

Il nous semble que ce dernier exemple illustre bien ce que l'on a dit au sujet de la relation entre langue générale et langue spécialisée, de la polysémie des mots, ainsi que de la présence des procédés métaphoriques dans la conceptualisation de nombreuses notions, non seulement dans le langage courant mais aussi dans la communication sectorielle, en le cas d'espèce économique (ce qui fera l'objet du paragraphe 3.4). Une fois les configurations sémantiques établies, le traducteur pourra vérifier s'il y a ou non une équivalence conceptuelle dans les deux langues, après quoi il choisira le traduisant le plus convenable.

\subsubsection{Termes complexes et polynotionnalité}

Il peut également arriver qu'une unité lexicale participe à des dénominations spécialisées ou termes complexes, chacun desquels renvoie à un concept bien précis selon le contexte (cf. Vandaele \& Raffo 2008). Tel est le cas, par exemple, de l'unité action que l'on repère dans plusieurs unités prédicatives (dont le type habituel est le verbe ou l'adjectif qualificatif), construites autour du sens central d'"acte, agissement" : 
a. initiatives ou projets dans le domaine de l'éducation (Erasmus)

17. Fr. Programme d'action communautaire en matière de mobilité des étudiants = It. Programma comunitario d'azione in materia di mobilità degli studenti

b. combat, lutte

18. Fr. Action politique / militaire / directe (grèves, manifestations) = It. Azione politica / militare / diretta

c. exercice d'un droit en justice (poursuite, recours)

19. Fr. Action mobilière / immobilière = It. Azione mobiliare / immobiliare

20. Fr. Action civile / pénale / légale = It. Azione civile / penale / legale

d. opération financière ou bancaire menée rapidement et visant à saisir une bonne occasion

21. Fr. Action éclair = It. Hit-and-run (operazione, azione lampo)

À remarquer ici la composante métaphorique du terme et l'emprunt anglais en italien.

e. en italien, trouble qui compromet la poursuite des débats (au sein de l'UE, lors de travaux parlementaires, ...)

22. Fr. Agitation = It. Azione di disturbo, tumulto

Les différents syntagmes terminologiques ci-dessus (action communautaire, action politique, action mobilière, etc.) constituent des termes spécifiques du générique action, dont l'acception de base ne change pas - il n'y a pas d'extensions de sens puisque l'unité garde la même signification lexicale - mais ce sont les notions tout à tour dénotées qui sont distinctes.

Dans la terminologie financière et comptable, le terme action désigne un titre, cessible et négociable, représentant une fraction du capital social (obligation, part, valeur) ; il s'agit là, pourtant, d'un cas d'homonymie, puisque le terme, employé avec cette signification, a un étymon distinct par rapport aux cas précédents, ce qui donne lieu à deux entrées dans le dictionnaire (1. déb. XII ${ }^{e}$, lat. actio [pour les signifiés présentés ci-dessus] ; 2. XVII" , probabl. de action, "dette active", par le holl. actie [pour le domaine financier]) (Le Petit Robert, 2012). En fait, de nombreuses études inhérentes à la construction du sens ont montré qu' 'il n'y a pas de coupure 
nette entre ces deux phénomènes" (Victorri \& Fuchs 1996 : 5), la distinction entre homonymie et polysémie relevant souvent d'un arbitraire lexicographique.

23. Fr. Action nominative / au porteur / titre d'action / certificat d'action = It. Azione nominativa / al portatore / titolo azionario / certificato azionario

24. Fr. Action à dividende prioritaire $(A D P)=$ It. Azione privilegiata (preferenziale)

25. Fr. Action en or $=$ It. Golden share

Ce dernier exemple confirme la tendance de la langue italienne à intégrer dans son vocabulaire les emprunts anglais attestés et légitimés au niveau international.

Ci-après, un autre cas de divergence entre le français et l'italien, relevant du fait que les cadres juridiques de référence ne sont pas tout à fait équivalents :

26. Fr. Société anonyme / S.A. / Soc. an. (le terme, désignant l'un des trois types de société par actions en France, est souvent utilisé comme générique de ladite catégorie) = It. Società per azioni / S.p.A. (le terme "società anonima" a été supprimé par le Code Civil italien de 1942, qui a introduit la distinction entre "società per azioni" et "società a responsabilità limitata")

En définitive, "l'équivalence interlinguistique des termes passe par la détermination de la notion" (Vandaele \& Raffo 2008 : 15) ainsi que par la connaissance du contexte - situationnel et culturel - qui connote et restreint le sens des termes.

\section{4. "Embodiment" et métaphores conceptuelles}

Lassociation entre un domaine conceptuel concret (par ex. canard $=$ animal) et un domaine abstrait (canard $=$ fausse nouvelle, canard boiteux $=$ entreprise instable, précaire) par l'intermédiaire du processus de métaphorisation nous offre un exemple emblématique du concept d'embodiment, envisageant que même les concepts abstraits, qui ne dérivent pas directement de l'expérience corporelle, y sont liés par des procédés imaginatifs, telle la métaphore qui permet d'ancrer la représentation mentale et l'expression linguistique d'un concept abstrait à un référent concret dérivant de l'expérience visuelle, 
motrice, etc. ${ }^{6}$ En d'autres termes, on projette la représentation d'un concept source (généralement concret ou, en tout cas, déjà connu, donc plus clair) sur un concept cible (plus complexe et difficile d'accès) pour faciliter la compréhension de celui-ci.

Plusieurs études, menées dans le cadre de la Sémantique Cognitive, ont souligné l'importance de la conceptualisation métaphorique dans la structuration du langage ; rappelons, à ce propos, les travaux de Vandaele en biomédecine et de Temmerman dans le domaine des sciences de la vie, ou encore de Mirowski sur la relation entre économie et physique, qui démontrent que "la compréhension de nouveaux domaines de recherche et les connaissances scientifiques se développent notamment par le biais du raisonnement métaphorique" (Temmerman 2007 : 72). Parallèlement, l'échange entre différents domaines spécialisés ou généraux, sous-jacent à la création des métaphores conceptuelles, se révèle un expédient essentiel pour l'évolution et l'enrichissement terminologique.

La langue de l'économie est imprégnée d'expressions métaphoriques systématiques (échelle mobile ; serpent monétaire / fiscal ; chute de la demande,...). Exploité surtout dans la presse de vulgarisation mais également présent dans les textes spécialisés, le mécanisme métaphorique permet de conceptualiser les notions économiques en termes d'entité physique, souvent animée et avec une orientation spatiale, ou bien, de patient, de bâtiment, de phénomène météorologique, etc. (la croissance des affaires fléchit ; marché en pleine convalescence; santé financière ; baromètre de confiance des investisseurs); on puise aussi dans les vocabulaires militaire, sportif, aéronautique, maritime (retraite prudente des investisseurs; décollage d'une entreprise ; l'économie a le vent en poupe). Évoquant des perceptions de la réalité, des schémas cognitifs propres à une communauté de parlants, les métaphores conceptuelles s'inscrivent dans les ICMs (Idealized Cognitive Models) qui dominent la vision culturelle d'un peuple (cf. Lakoff \& Johnson 1980, Lakoff 1987).

Sans entrer dans les détails des typologies de métaphores économiques analysées en termes de projection inter-domaniale, conformément à l'approche cognitive (pour un aperçu de ce sujet, nous renvoyons à Rollo 2012), nous nous limiterons ici à présenter quelques cas de figures en ce qui concerne les modalités de traduction du français à l'italien. S'agissant de deux langues

6. Les linguistes cognitifs font une distinction terminologique entre métaphore, soit l'activité cognitive proprement dite, l'opération de mapping entre deux domaines ou cadres conceptuels, et expression métaphorique, c'est-à-dire l'occurrence verbale, la réalisation linguistique de structures mentales sous-jacentes, elles-mêmes métaphoriques. 
assez proches du point de vue socioculturel, les associations épistémiques et ontologiques entre différents domaines conceptuels sont dans leurs grandes lignes les mêmes, ce qui accroît le degré de traduisibilité ; il n'en reste pas moins que certaines expressions présentent des configurations idiosyncrasiques, renvoyant à des projections conceptuelles particulières à l'une ou à l'autre langue, elles demandent, par conséquent, la mise en œuvre de stratégies différentes pour leur traduction.

- Traduction littérale: Métaphore $\rightarrow$ Métaphore (même domaine source et même image)

27. Fr. Les prix ont grimpé / Le titre dégringole de 25,5\% à la mi-journée $=$ It. I prezzi hanno avuto un'impennata / Il titolo crolla (precipita) del $25,5 \%$ a metà giornata

28. Fr. Renflouer / Remettre sur pied une entreprise = It. $\underline{\text { Rimettere in piedi }}$ un'azienda

29. Fr. La très grande souplesse de la politique monétaire a ranimé l'inflation = It. L'estrema flessibilità della politica monetaria ha rianimato l'inflazione

30. Fr. Le baromètre de l'économie affiche des indicateurs toujours dans le $\underline{\text { rouge }}=$ It. La Il barometro dell'economia mostra indicatori sempre $\underline{\text { in }}$ $\underline{\text { rosso }}$

31. Fr. Changement de cap de la Banque nationale suisse = It. Inversione di rotta della Banca nazionale svizzera

- Modulation métaphorique :

a. Métaphore - $_{1}->$ Métaphore $_{2}$ (même domaine source, mais image différente)

32. Fr. L'économie avance à pas de tortue = It. L'economia avanza a passo di lumaca

33. Fr. Les effectifs salariés de Carrefour ont très légèrement reculé en Europe [orientation spatiale : sens "avant-derrière"] = It. Lorganico di dipendenti di Carrefour è leggermente calato in Europa [sens "dessus-dessous"]

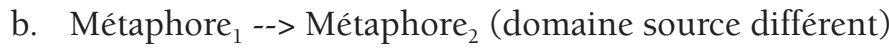




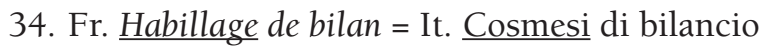

- Adaptation : Métaphore lexicalisée / Expression figée => Locution / Expression figée propre à la langue cible

35. Fr. Moral des investisseurs en berne $=>$ It. Morale degli investitori in $\underline{\text { crisi }}$

36. Fr. Le syndicat CFE-CGC tire à boulet rouge sur sa direction et la nouvelle stratégie de l'opérateur = It. Il sindacato CFE-CGC spara a zero sulla direzione e la nuova strategia dell'operatore

- Mise à zéro : Métaphore $=>\varnothing$ Pas de métaphore (et vice-versa)

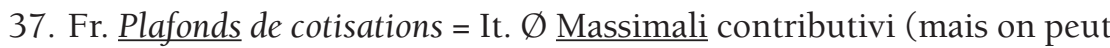
garder la même métaphore en traduisant plafond par "tetto massimo")

38. Fr. $\emptyset$ Système bancaire parallèle $=$ It. Sistema bancario ombra

Il est indiscutable que les expressions métaphoriques concourent à rendre le discours économique plus vif et efficace, en focalisant certaines propriétés saillantes d'un concept et en en celant d'autres. Du point de vue traductif, on constate qu'il y a souvent une parfaite symétrie entre le français et l'italien (représentée avec une flèche continue), surtout pour les métaphores qui ont la même fréquence d'emploi dans les deux langues et qui puisent dans les mêmes domaines sources. Néanmoins, il y a des cas où le traducteur ne peut pas récréer le même scénario métaphorique que la langue source, quitte à produire des images inadéquates en langue cible; il est donc obligé d'intervenir par une modulation (flèche discontinue) ou une adaptation, voire une mise à zéro de la métaphore (passage d'un frame métaphorique à un frame non métaphorique), face à l'absence d'une expression correspondante dans la langue d'arrivée.

Lapproche conceptuelle prônée par les linguistes cognitifs peut s'avérer un atout précieux lors du transfert traductif, parce qu'elle permet de déterminer si les expressions employées dans les langues en jeu renvoient aux mêmes domaines source et cible ou si les images utilisées pour représenter un concept n'ont pas la même charge dénotative et connotative, ce qui pourra aider le traducteur à aboutir à un résultat sémantiquement et pragmatiquement équivalent. 


\subsection{Contexte de la communication et strates de spécialisation}

Les divers niveaux de spécialisation sont eux-mêmes déclinés suivant les destinataires (experts, non-experts, néophytes) et les besoins de communication - c'est ce que l'on appelle la "dimension verticale" ou pragmatique des langues spécialisées (Cortelazzo 1990); il ressort de là l'exigence d'évaluer le contexte communicatif en fonction duquel la langue doit être modulée.

Dans un texte spécialisé prototypique, la terminologie technique prolifère et plusieurs concepts sont donnés pour acquis, mais c'est précisément le haut degré de technicité et de formalisme qui assure une parfaite transparence chez les experts du secteur ; autrement dit, une terminologie spécialisée sera d'autant plus claire que le niveau de compétence et de culture générale du public final sera plus élevé. Par contre, les textes à visée vulgarisatrice, visant à transmettre des connaissances professionnelles au grand public, imposent le recours à un langage aussi intelligible que possible, accompagné de notes explicatives ou de parenthèses s'il y a lieu, ainsi que d'une réorganisation structurelle du texte ; il y a aussi des mots apparemment synonymiques mais qui diffèrent quant à leur statut et à leur fonction pragmatique.

En tant qu'acte de communication, la traduction économique se fonde donc sur une série de choix et de stratégies qu'il faut déterminer au cas par cas selon la situation spécifique, définie à son tour par la finalité du texte traduit, par les caractéristiques des destinataires, par la culture et la sensibilité linguistique du traducteur (cf. Faini 2008 : 32-33). Celui-ci peut juger opportun de traduire différemment certains termes ou expressions, en optant tantôt pour des solutions formelles et articulées, tantôt pour des tournures plus claires et explicites.

Ci-après, deux extraits tirés du Rapport du Conseil des prélèvements obligatoires. Impôt sur le revenu, CSG quelles réformes? (février 2015) dont nous proposons d'abord une traduction gardant le même niveau de spécialité que le texte source, ensuite, une traduction explicitante, avec l'emploi d'une terminologie plus pertinente à un contexte de divulgation, dans le but d'éclaircir quelques notions relatives à la fiscalité, notamment du système français. 


\begin{tabular}{|c|c|}
\hline FR & IT \\
\hline \multirow[t]{2}{*}{$\begin{array}{l}\text { Une surtaxe progressive, chargée " d'ajuster } \\
\text { la charge fiscale à la capacité contributive } \\
\text { de chacun, cela par le jeu de l'abattement } \\
\text { à la base, du quotient familial et du taux } \\
\text { progressif ». La surtaxe frappait le montant } \\
\text { total du revenu net global et comportait } \\
\text { un barème à neuf tranches [...]. (p. 20) }\end{array}$} & $\begin{array}{l}\text { Una sovrattassa progressiva, volta } \\
\text { all'«adeguamento della pressione } \\
\text { fiscale alla capacità contributiva di } \\
\text { ciascuno, attraverso il meccanismo } \\
\text { dell'abbattimento alla base, del quoziente } \\
\text { familiare e dell'aliquota progressiva». La } \\
\text { sovrattassa colpiva l'ammontare totale del } \\
\text { reddito netto complessivo e comportava } \\
\text { un listino / una tabella contributiva a nove } \\
\text { scaglioni [...]. }\end{array}$ \\
\hline & $\begin{array}{l}\text { Una sovrattassa progressiva, volta } \\
\text { all'«adeguamento della pressione } \\
\text { fiscale alla capacità contributiva di } \\
\text { ciascuno, attraverso il meccanismo } \\
\underline{\text { della detrazione / dello sgravio fiscale }} \\
\text { alla base, del quoziente familiare } \\
\text { dell'aliquota progressiva». La sovrattassa } \\
\text { colpiva l'ammontare totale del reddito } \\
\text { netto complessivo e comportava una } \\
\underline{\text { tabella esattoriale / tabella delle aliquote }} \\
\underline{\text { contributive a nove fasce [...]. }} \\
\underline{\underline{1} \text { Strumento adottato nel modello di }} \\
\underline{\text { imposizione fiscale francese, che consente }} \\
\underline{\text { di calcolare l'imposta sul reddito in }} \\
\underline{\text { funzione delle persone fiscalmente a }} \\
\underline{\text { carico. }}\end{array}$ \\
\hline $\begin{array}{l}\text { Au sein des salariés ensuite, les différences } \\
\text { de taux de cotisations, les assiettes } \\
\text { dérogatoires pour certaines catégories de } \\
\text { salariés ou encore les règles de déduction } \\
\text { représentative des frais professionnels } \\
\text { aboutissent à des effets variables pour un } \\
\text { même niveau d'IR et au sein même de chaque } \\
\text { catégorie de salariés. [...] } \\
\text { Entre les non-salariés enfin, compte tenu } \\
\text { des différences de règles de plafonnement et } \\
\text { de cotisation minimale, de taux ou de mode } \\
\text { de calcul des cotisations forfaitaires, tout } \\
\text { alignement d'assiette aurait également des } \\
\text { conséquences variables entre travailleurs } \\
\text { indépendants, rendant impossible le recours } \\
\text { à un mécanisme de correction uniforme. (p. } \\
\text { 147) }\end{array}$ & $\begin{array}{l}\text { Tra i lavoratori dipendenti, poi, le } \\
\text { differenze di aliquota contributiva, le } \\
\text { basi imponibili derogatorie per talune } \\
\text { categorie di lavoratori subordinati } \\
\text { o ancora le regole di deduzione } \\
\text { rappresentativa delle spese professionali } \\
\text { raggiungono effetti variabili per uno } \\
\text { stesso livello di IR nonché all'interno di } \\
\text { ogni categoria di lavoratori dipendenti. } \\
\text { [...] } \\
\text { Infine, tra i lavoratori autonomi, tenuto } \\
\text { conto delle diverse regole di massimale } \\
\text { e minimale contributivo, di aliquota o } \\
\text { modalità di calcolo dei contributi forfetari, } \\
\text { ogni adeguamento della base imponibile } \\
\text { avrebbe parimenti effetti variabili } \\
\text { tra lavoratori autonomi, rendendo } \\
\text { impossibile il ricorso a un meccanismo } \\
\text { correttivo uniforme. }\end{array}$ \\
\hline
\end{tabular}




\begin{tabular}{|c|c|c|}
\hline & & $\begin{array}{l}\text { Tra i lavoratori dipendenti, poi, le } \\
\text { differenze di aliquota contributiva [...] } \\
\text { raggiungono effetti variabili per uno } \\
\text { stesso livello di IR (imposta sul reddito) } \\
\text { nonché all'interno di ogni categoria di } \\
\text { lavoratori dipendenti. [...] } \\
\text { Infine, tra i lavoratori autonomi, tenuto } \\
\text { conto delle diverse regole di massimale } \\
\underline{\text { e minimale contributivo }}{ }^{2} \text {, di aliquota o } \\
\text { modalità di calcolo dei contributi forfetari, } \\
\text { ogni adeguamento della base imponibile } \\
\text { avrebbe parimenti effetti variabili tra } \\
\text { lavoratori autonomi [...]. } \\
\underline{2} \text { Tetto massimo e minimo dei contributi } \\
\text { che i lavoratori devono versare. }\end{array}$ \\
\hline
\end{tabular}

Il faut également tenir compte que, même dans une situation analogue, deux langues peuvent employer des variantes lexico-terminologiques et des structures grammaticales différentes, comme l'illustrent les deux exemples ci-dessous, repérés sur le site de "Banca Popolare di Puglia e Basilicata", dans une brochure plurilingue - Strumenti e servizi bancari, dalla A alla $Z$ - destinée aux ressortissants étrangers qui vivent et travaillent en Italie, pour leur illustrer les outils et les services offerts par la banque en vue de choix plus éclairés.

\begin{tabular}{|c|c|}
\hline IT & FR \\
\hline $\begin{array}{l}\text { AVERE UN PRESTITO - La banca non } \\
\text { dà automaticamente un prestito a chi lo } \\
\text { chiede. Prima di dare una carta di credito, } \\
\text { un prestito, un mutuo, la banca fa una } \\
\text { valutazione creditizia, cioè verifica se } \\
\text { il cliente ha la possibilità di restituire i } \\
\text { soldi nel tempo. Più la banca conosce } \\
\text { la persona che chiede il prestito, più ha } \\
\text { informazioni per poter valutare il cliente, } \\
\text { e più è possibile che la banca gli presti dei } \\
\text { soldi. Se il cliente ha emesso un assegno } \\
\text { senza avere i soldi disponibili, sarà molto } \\
\text { difficile ottenere un prestito. [...] (p. 8) }\end{array}$ & $\begin{array}{l}\text { OBTENIR UN PRÊT - La banque n'accorde } \\
\text { pas automatiquement de prêts à toutes les } \\
\text { personnes qui en font la demande. Avant } \\
\text { de remettre une carte de crédit, d'accorder } \\
\text { un prêt, un emprunt, la banque évalue la } \\
\text { solvabilité du demandeur et vérifie donc } \\
\text { s'il a la possibilité de lui restituer l'argent } \\
\text { dans le temps. Plus la banque connaît le } \\
\text { demandeur, plus elle a d'informations pour } \\
\text { évaluer sa situation et plus il est probable } \\
\text { qu'elle lui prête de l'argent. Si le client a } \\
\text { émis un chèque sans provision, il lui sera } \\
\text { très difficile d'obtenir un prêt. [...] (p. 24) }\end{array}$ \\
\hline
\end{tabular}

Notons avant tout qu'en correspondance de certaines formes verbales "passe-partout" du texte italien ("avere", "dare", "fare"), plus fréquentes dans les documents destinés au grand public, on emploie dans la traduction française des structures argumentales prédicatives diversifiées, où la base de la collocation se combine à un collocatif verbal bien précis, dont le sens se construit dans le contexte de la base : "Avere un prestito" = Obtenir un 
prêt ; "La banca dà un prestito / un mutuo / una carta di credito" = La banque accorde un prêt, un emprunt ${ }^{7}$ / remet une carte de crédit; "La banca fa una valutazione creditizia" = La banque évalue la solvabilité du demandeur. De plus, la construction nominale se confirme un frame particulièrement récurrent en français, susceptible de condenser l'information exprimée, alors que l'italien est un peu plus enclin à souligner le mouvement du processus, la trajectoire de la "figure" ou entité proéminente : "la persona che chiede il prestito" = le demandeur ; "senza avere i soldi disponibili" = sans provision.

\begin{tabular}{|c|c|}
\hline IT & FR \\
\hline $\begin{array}{l}\text { Bancomat } \\
\text { Per avere una carta Bancomat, bisogna } \\
\text { aprire un conto corrente. In Italia con il } \\
\text { Bancomat può prelevare soldi agli sportelli } \\
\text { automatici (ATM) e pagare nei negozi che } \\
\text { hanno il terminale vicino alla cassa se il } \\
\text { Bancomat ha la scritta Pagobancomat. Se } \\
\text { la carta è collegata a circuiti internazionali } \\
\text { come Visa Electron, Cirrus, Maestro, } \\
\text { può pagare gli acquisti nei negozi anche } \\
\text { all'estero. } \\
\text { Con il Bancomat può anche verificare il } \\
\text { saldo e i movimenti del conto corrente, } \\
\text { ricaricare il cellulare, fare versamenti, } \\
\text { prelevare o pagare fino a una cifra } \\
\text { massima mensile. Quando paga o preleva, } \\
\text { le vengono immediatamente tolti i soldi } \\
\text { dal conto corrente: per questo il Bancomat } \\
\text { si chiama carta di debito. Per usare il } \\
\text { Bancomat, deve digitare il codice segreto } \\
\text { numerico della carta (PIN). (p. } 12 \text { ) }\end{array}$ & $\begin{array}{l}\text { Carte Bancomat } \\
\text { Pour avoir une carte Bancomat, il faut } \\
\text { ouvrir un compte courant. En Italie, la carte } \\
\text { Bancomat permet de retirer de l'argent aux } \\
\text { distributeurs automatiques (ATM) et de } \\
\text { payer des achats dans les magasins équipés } \\
\text { d'un terminal près de la caisse si la carte } \\
\text { Bancomat porte la mention PagoBancomat. } \\
\text { Si la carte est reliée aux circuits } \\
\text { internationaux comme Visa Electron, Cirrus, } \\
\text { Maestro, il est possible de régler dans les } \\
\text { magasins les achats effectués à l'étranger. } \\
\text { Avec la carte Bancomat, il est également } \\
\text { possible de vérifier le solde et les } \\
\text { mouvements d'un compte courant, de } \\
\text { recharger un téléphone portable, d'effectuer } \\
\text { des versements ou de payer mensuellement } \\
\text { un montant maximum. Lorsque vous payez } \\
\text { ou retirez de l'argent, le montant demandé } \\
\underline{\text { est immédiatement débité du compte }} \\
\text { courant : c'est la raison pour laquelle la } \\
\text { carte Bancomat est appelée carte de débit. } \\
\text { Pour utiliser la carte Bancomat, il faut } \\
\text { saisir le code numérique secret de la carte } \\
\text { (PIN). (pp. 28-29) }\end{array}$ \\
\hline
\end{tabular}

Le scénario ici décrit est celui de l'emploi d'une carte Bancomat, avec une série de situations y associées : retrait d'argent aux distributeurs automatiques, achats et paiements sans argent liquide (jusqu'à un montant maximum mensuel), vérification du solde, débit sur le compte courant, saisie d'un code secret. Les changements traductifs principaux lors du passage de l'italien au français concernent l'alternance entre forme personnelle et forme

7. Dans ce guide on trouve aussi : Avere un mutuo = Faire un emprunt $;$ Mutuo (en tant que service offert pas la banque) $=$ Prêt immobilier . 
impersonnelle et l'asymétrie de la relation figure/ground, avec un focus différent sur les concepts proéminents. Conformément aux conventions respectées dans ce type de document, la version italienne tend à mettre en avant la "figure" du client, soit le possesseur de la carte qui se distingue du "fond" (emploi du pronom personnel sujet de politesse "lei", toujours sous-entendu, et du pronom personnel complément "le" : "può prelevare", "può pagare", "le vengono tolti"...), tandis que le français privilégie la construction impersonnelle avec le pronom il (il est possible de...) ou la voix passive sans complément d'agent (le montant demandé est débité...) ; de même, c'est le service offert (la carte) qui est mis en relief en français comme "figure" et sujet grammatical du deuxième énoncé (La carte Bancomat permet de retirer de l'argent), là où en italien il fait fonction de complément circonstanciel ("con il Bancomat può prelevare soldi").

Tout en respectant la structure fondamentale de l'original, le texte d'arrivée présente donc de légers changements de perspective qui s'actualisent sur le plan verbal, ce qui prouve, une fois de plus, que la structure linguistique reflète l'organisation conceptuelle sous-jacente.

\section{Pour conclure}

Passage obligé des échanges et des transactions internationales dans un marché de plus en plus compétitif et performant, la traduction économique se configure comme un travail spécialisé exigeant la mise en œuvre de connaissances approfondies et bien assises à plusieurs niveaux : linguistique, terminologique, technique, traductologique, culturel. Convoquant diverses disciplines, elle ne peut pas faire abstraction de la prise en compte de notions théoriques qui vont nourrir et soutenir la pratique traductive, à côté des outils terminographiques et des traitements automatiques.

C'est là l'aspect central de cette étude où nous avons cherché à démontrer, à l'aide de quelques exemples pratiques, l'importance d'une réflexion théorique dans la formation et dans l'activité du traducteur ; comme le souligne Guidère (2010 : 117), "la formation à la spécialisation est devenue un cadre englobant qui relie le monde académique au monde professionnel". En particulier, nous avons vu comment certains concepts issus de la Linguistique et de la Sémantique Cognitive peuvent concourir à décortiquer les textes et à en cerner les particularités conceptuelles et traductives, de sorte que le traducteur puisse gérer et résoudre au mieux les enjeux qu'ils posent.

En conclusion, nous souhaitons qu'à l'avenir les contributions théoriques et les études menées dans le domaine académique trouvent application dans le marché de la traduction spécialisée, en l'occurrence économique, et que les 
professionnels du secteur puissent s'appuyer à la fois sur l'expérience acquise sur le terrain et sur des outils théoriques solides et fiables, qui leur permettent de surmonter les nombreuses difficultés survenant dans l'exercice de leur métier.

\section{Bibliographie}

BÉJOINT, Henri \& Philippe Thoiron. (1996) "Introduction." In: Béjoint, Henri \& Philippe Thoiron (éds.) 1996. Les dictionnaires bilingues. Oxford/New York: De Boeck Supérieur, pp. 5-15.

BEUCHAT, Alice. (2014) La traduction économique. Théorie et pratique. Saarbrücken: Éditions universitaires européennes.

CABRÉ, M. Teresa. (2010) "Terminology and translation". In: Gambier, Yves \& Luc Van Doorslaer (eds.) 2010. Handbook of Translation Studies, vol. 1. Amsterdam/Philadelphia: John Benjamins Publishing Company, pp. 356-365.

ClAS, André. (2005) "Éditorial" Meta $50: 1$, pp. 1-5. Version électronique : <https://www.erudit.org/revue/meta/2005/v50/nl/01065lar.pdf>

Cortelazzo, Michele. (1994) Lingue speciali. La dimensione verticale, 2a ed. Padova: Unipress.

DANCETTE, Jeanne \& Sonia Borotra. (1997) "Quasi synonymie et non-isomorphie dans le dictionnaire spécialisé bilingue." In : Kocourek, Rostislav (éd.) 1997. Actes de langue française et de linguistique 8/9. Halifax, pp. 63-79.

DANCETTE, Jeanne \& Sonia Halimi. (2005) "La représentation des connaissances ; son apport à l'étude du processus de traduction." Meta $50: 2$, pp. 548-559. Version électronique : <http://www.erudit.org/revue/meta/ 2005/ v50/n2/010999ar.pdf>

DANCETTE, Jeanne. (1992) "La complexité de la langue économique et commerciale au Québec, les défis du traducteur." Terminologie et Traduction 2 : 3, pp. 197-210.

DANCETTE, Jeanne. (1995) "Organisation conceptuelle du domaine et structure de dictionnaire - L'exemple du commerce de détail." TTR ${ }^{\circ}$ : traduction, terminologie, rédaction $8: 2$, pp. 151-174. Version électronique : <https://www. erudit.org/revue/ttr/1995/v8/n2/03722lar. pdf>

DANCETTE, Jeanne. (2003) "Lélaboration de la cohérence en traduction; le rôle des référents cognitifs." TTR ${ }^{\circ}$ : traduction, terminologie, rédaction $16: 1$, pp. 141-159. Version électronique : <http://www.erudit.org/revue/ttr/2003/v16/ n1/008560ar.pdf>

DELBECQUE, Nicole (éd.) (2002) Linguistique cognitive. Comprendre comment fonctionne le langage. Bruxelles : De Boeck-Duculot.

FABER, Pamela \& José Manuel Ureña Gómez-Moreno. (2012) "Specialized language translation." In: Faber, Pamela (ed.) 2012. A Cognitive Linguistics View 
of Terminology and Specialized Language. Berlin/Boston: De Gruyter Mouton, pp. 73-92.

FAINI, Paola. (2008) Tradurre. Manuale teorico e pratico. Roma: Carocci.

FILlmore, Charles J. \& Beryl T. Atkins. (1992) "Toward a frame-based lexicon:

The semantics of RISK and its neighbours". In: Lehrer, Adrienne \& Eva

Kittay (eds.) 1992. Frames, fields, and contrasts. Hillsdale/N.J.: Lawrence ErlbaumAssoc, pp. 75-102.

FILlmore, Charles J. (1977) "Scenes-and-frames semantics." In: Zampolli, Antonio (ed.) 1977. Linguistic Structures Processing. Amsterdam/New York: North Holland Publishing Company, pp. 55-81.

Fillmore, Charles J. (1985) "Frames and the Semantics of Understanding." Quaderni di Semantica IV, pp. 217-240.

Gallego Hernández, Daniel. (2010) "Estudio comparativo trilingüe de la traducción de la metáfora náutica en el lenguaje económico-financiero." In: Mogorrón Huerta, Pedro \& Salah Mejri (eds.) 2010. Opacité, idiomaticité, traduction. Alicante: Universidad de Alicante, pp. 99-128.

GEERAERTS, Dirk \& Hubert Cuyckens. (2007) "Introducing Cognitive Linguistics." In: Geeraerts, Dirk \& Hubert Cuyckens (eds.) 2007. The Oxford Handbook of Cognitive Linguistics. Oxford/New York: Oxford University Press, pp. 3-21.

GÉMAR, Jean-Claude. (2007) "Retour à la sagesse ? Sept piliers du savoir-faire du traducteur juridique." In : Lavault-Olléon, Élisabeth (éd.) 2007. Traduction spécialisée : pratiques, théories, formation. Bern: Peter Lang, pp. 27-43.

Guidère, Mathieu. (2010) Introduction à la traductologie. Penser la traduction: hier, aujourd'hui, demain. Bruxelles : De Boeck Université.

KleIBER, Georges. (1990) La sémantique du prototype. Catégories et sens lexical. Paris : PUF.

LACROIX, Kim. (2010) "Le traducteur et la recherche terminologique ponctuelle au $21^{\mathrm{e}}$ siècle." Actualité langagière, vol. 7/4 [En ligne]. Version électronique : <http://www.btb.termiumplus.gc.ca/tpv2guides/guides/chroniq/index-fra. html? lang=fra\&lettr=indx_autr8Oz22dTiL13Y\&page=9EFNIlrlH4zg.html>

LAKOFF, George \& Mark Johnson. (1980) Metaphors We Live By. Chicago: The University of Chicago Press. Cité par la traduction en français de Michel de Fornel en collaboration avec Jean-Jacques Lecercle : Les métaphores dans la vie quotidienne. Paris : Les Éditions de Minuit, 1985.

LAKOFF, George. (1987) Women, Fire and Dangerous Things. What categories reveal about the Mind. Chicago and London: The University of Chicago Press.

LAVAUlT-OlLÉON, Élisabeth. (2007) "Traduction spécialisée : des pratiques qui se passent de théorie ?" In : Lavault-Olléon, Élisabeth (éd.) 2007. Traduction spécialisée : pratiques, théories, formation. Bern: Peter Lang SA, pp. 45-71.

LE BRIS, Anne. (2001) L'économie et les affaires, Dizionario fraseologico francese-italiano italiano-francese. Bologna: Zanichelli. 
LERAT, Pierre. (1995) Les langues spécialisées. Paris : PUF.

LEWANDOWSKA, Barbara \& Marcel Thelenand Tomaszczyk (eds.) (2002) Translation and meaning, Proceedings of the Maastricht Session of the 3rd International Maastricht-Lódz Duo Colloquium on "Translation and Meaning", held in Lódz, Poland, 22-24 September 2000. Maastricht: Universitaire Pers Maastricht.

MEjRI, Salah. (2005) "Traduire, c'est gérer un déficit." Meta 50 : 1, pp. 120128. Version électronique : <https://www.erudit.org/revue/meta/2005/v50/nl/ 010662ar.pdf>

RESCHE, Catherine. (2002) "La métaphore en langue spécialisée, entre médiation et contradiction : étude d'une mutation métaphorique en anglais économique." Asp 35-36 [En ligne], pp. 103-119. Version électronique : <http://asp. revues.org/1624>

ReY-Debove, Josette \& Alain Rey (dir.). (2012) Le Petit Robert. Dictionnaire alphabétique et analogique de la langue française, Nouvelle édition du Petit Robert. Paris : Le Robert.

Rollo, Alessandra. (2008) "Interculturalité, Traductologie et Linguistique Cognitive." Lingue e Linguaggi 1, Lecce: Pensa MultiMedia Editore, pp. 51-71.

Rollo, Alessandra. (2012) "Les métaphores dans le lexique économique : modèles culturels en œuvre.” In: Ligas, Pierluigi \& Paolo Frassi (éds.) 2012. Lexiques Identités Cultures. Verona : Qui Edit, pp. 153-175.

Rollo, Alessandra. (à paraître) "Les enjeux culturels de la traduction économique français-italien-français : choix traductologiques et stratégies en œuvre." Colloque Impliciter, expliciter. Le traducteur comme équilibriste interculturel, Université de Lièges \& Haute Ecole de la ville de Liège, 2-4 mai 2013.

SERÓN ORdóÑEZ, Inmaculada (2005) "La traducción de la metáfora en los textos financieros: estudio de caso." In: Gracia Torres, M. ${ }^{a}$ (ed.) 2005. Traducción y cultura. El referente cultural en la comunicación especializada, Málaga: Encasa, pp. 205-250.

SElESKOVITCH, Danica \& Marianne Lederer. (2001) Interpréter pour traduire, $4^{\mathrm{e}}$ édition revue et corrigée (1ère éd. 1984). Paris : Didier Érudition.

SnELL-Hornby, Mary. (1995) Translation Studies. An integrated approach, Revised Edition (1st ed. 1988). Amsterdam/Philadelphia: John Benjamins.

TALmy, Leonard. (1978) "Figure and Ground in Complex Sentences." In: Greenberg, Joseph et al. (eds.) 1978. Universals of human language 4. Stanford: Stanford University Press, pp. 625-649.

Temmerman, Rita. (2000) Towards New Ways of Terminology Description. The sociocognitive approach. Amsterdam/Philadelphia: John Benjamins Publishing Company. 
Temmerman, Rita. (2007) "Les métaphores dans les sciences de la vie et le situé socioculturel." Cahiers du Rifal 26, pp. 72-83. Version électronique : <http:// www.rifal.org/cahiers/rifal26/crf-26-07.pdf>

Tholron, Philippe \& Henri Béjoint. (2010) "La terminologie, une question de termes?" Meta 55 : 1, pp. 105-118. Version électronique : <https://www.erudit.org/revue/meta/2010/v55/n1/039605ar.pdf>

VANDAElE, Sylvie \& Leslie Lubin. (2005) "Approche cognitive de la traduction dans les langues de spécialité : vers une systématisation de la description de la conceptualisation métaphorique." Meta 50 : 2, pp. 415-431. Version électronique : <http://www.erudit.org/revue/meta/2005/v/n2/ 010991ar.html>

VANDAElE, Sylvie \& Mariana Raffo. (2008) "Significations lexicales et notionnelles dans les domaines de spécialité." Festschrift en l'honneur d'Henri Béjoint, Université Lumière Lyon-2, Lyon. Version électronique : <https://papyrus.bib. umontreal.ca/xmlui/bitstream/ handle/1866/2598/VandaeleRaffoSign.pdf? sequence $=1>$

VICTORRI, Bernard \& Catherine Fuchs. (1996) La polysémie - construction dynamique du sens. Hermès. Version électronique : <https://halshs.archivesouvertes.fr/halshs-00713735>

VINTI, Claudio. (2006) "La métaphore dans la presse économique italienne : quelques réflexions." In: Benelli, Graziano \& Giampaolo Tonini (eds.) 2006. Studi in ricordo di Carmen Sánchez Montero. Trieste: EUT Edizioni Università di Trieste, vol. 2, pp. 557-567. Version électronique : <http://www.openstarts. units.it/dspace/bitstream/10077/7966/4/Vinti.pdf>

Sitographie

$<$ http://europa.eu/pol/index_fr.htm>

$<$ http://www.bppb.it/abi-benvenuto-in-banca>

$<$ http://www.cleiss.fr/docs/regimes/regime_france.html>

$<$ http://www.economie.gouv.fr/cedef/veille-documentaire-banque-finances>

$<$ https://www.ccomptes.fr/Institutions-associees/Conseil-des-prelevementsobligatoires-CPO3>

$<$ http://www.ilsole24ore.com/>

$<$ http://www.latribune.fr/>

Dictionnaires en ligne

$<$ http://atilf.atilf.fr>

$<$ http://iate.europa.eu>

$<$ http://www.franceterme.culture.fr> Vocabulaire de l'économie et des finances. Enrichissement de la langue française. (2012) Termes, expressions et définitions 
publiés au Journal officiel, Premier ministre, Commission générale de terminologie et de néologie.

<http://zedamt.herokuapp.com/> Dancette, Jeanne. (2013) Dictionnaire analytique de la mondialisation et du travail (DAMT). Multilingue anglais-espagnolfrançais.

$<$ http://www.lexilogos.com/dictionnaire_commercial.htm>

$<$ http://www.trader-finance.fr/lexique-finance/s

$<$ http://lexique-financier.actufinance.fr/>

\section{BIONOTE /NOTICE BIOGRAPHIQUE}

Alessandra Rollo is a Tenured Researcher of French Language and Translation at the University of Salento. In 2005 she received her Ph.D. in French Linguistics from the University of Salento; the subject of her dissertation was Cognitive Linguistics. In 2007 she attended a Distance Specialization Course in "Specialized economic, banking and financial translation". Her main research interests are in the areas of: Cognitive Linguistics and its implications in the domains of pragmatics and translation; specialized translation (mainly economic and multimedia fields). In addition to a regular didactic activity at the University of Salento, she has held some courses in postgraduate Masters. She has published three volumes (La Linguistica Cognitiva: dalle teorie alla grammatica, 2004, Regards sur le français contemporain, 2009, Bande dessinée et jeux de mots. Enjeux et stratégies traductives dans la traduction italienne d'“Astérix", 2012), besides other articles on French language and linguistics.

Alessandra Rollo est enseignante-chercheuse en Langue et Traduction françaises à l'Université du Salento. Elle est titulaire d'un Doctorat en Linguistique française (soutenance de thèse en Linguistique Cognitive, Université du Salento, 2005). En 2007 elle a suivi un Cours de perfectionnement à distance en "Traduction spécialisée dans le domaine de l'économie, de la banque et de la finance". Ses champs d'intérêt comprennent entre autres: la Linguistique Cognitive et ses implications dans les domaines pragmatique et traductologique; la traduction spécialisée, notamment dans les secteurs économique et audiovisuel. En plus d'une activité didactique régulière à l'Université du Salento, elle a donné des cours de 3ème cycle. Elle a publié trois monographies (La Linguistica Cognitiva: dalle teorie alla grammatica, 2004; Regards sur le français contemporain, 2009; Bande dessinée et jeux de mots. Enjeux et stratégies traductives dans la traduction italienne d'“Astérix", 2012), à côté de nombreux articles de langue et linguistique françaises. 
